\title{
The Probiotic Properties of Lactobacilli in Organic Pigs
}

\author{
D. Liu ${ }^{\mathrm{a}}$, K. Direksin ${ }^{\mathrm{a}, *}$, \& M. Panya ${ }^{\mathrm{b}}$ \\ aDivision of Livestock Medicine, Faculty of Veterinary Medicine, Khon Kaen University, \\ 123 Mittraparp Road, Muang District, Khon Kaen, 40002, Thailand \\ ${ }^{\mathrm{b} C o l l e g e}$ of Medicine and Public Health, Ubon Ratchathani University \\ 85 Sathollmark Road, Warinchamrap, Ubon Ratchathani, 34190, Thailand \\ *Corresponding author: kochakrn@kku.ac.th \\ (Received 15-06-2021; Revised 25-07-2021; Accepted 16-08-2021)
}

\begin{abstract}
Indigenous Lactobacilli are suitable probiotics because they adapt well in the hosts and ecological niches. Here we test local Lactobacillus for future application in the pigs as the farm-autogenous strains. The objectives of this study were to evaluate the probiotic properties of Lactobacillus isolated from the feces of antibiotic-free organic pigs. The properties include bile salt and pepsin tolerance, survival in storage $\left(37 \& 4{ }^{\circ} \mathrm{C}\right)$ and probiotic-packaging $\left(50{ }^{\circ} \mathrm{C}\right)$ temperatures, antibiogram, and antagonistic activity against Salmonella typhimurium ATCC 13311 and Escherichia coli ATCC 25922. Eighteen isolates with three different species were tested in this study as follows: L. reuteri (seven strains), L. mucosae (ten strains), and L. plantarum (one strain). Four isolates - L. reuteri-OP1, L. mucosae$\mathrm{OP2}$, L. mucosae-OP3, and L. reuteri-OP17-had good in vitro probiotic characteristics. Eleven isolates completely inhibited both E. coli and S. typhimurium. The other isolates are perfectly disabled, either E. coli or S. typhimurium. Despite that, they caused a reduction in the numbers of each pathogen. All Lactobacilli tested were susceptible to amoxicillin-clavulanate, ampicillin, and imipenem. Most isolates were sensitive to clindamycin $(72 \%)$, gentamicin $(56 \%)$, and tetracycline $(50 \%)$. Half of the proportions were somewhat sensitive/resistant to cefotaxime $(39 / 44 \%)$, tetracycline $(50 / 39 \%)$, and streptomycin $(39 / 56 \%)$. One hundred percent of Lactobacilli were resistant to norfloxacin, sulfamethoxazole-trimethoprim, and vancomycin, while $94 \%$ were resistant to enrofloxacin. Most of the local Lactobacilli passed in vitro tests, but the efficacy of probiotics in pigs awaits further in vivo investigation. Therefore, the potential probiotic strains derived from this study could be selected for further evaluation of their probiotic roles in economic pigs.
\end{abstract}

Keywords: antibiotic-free pig; antibiotic resistant; Escherichia coli; farm-autogenous strain; indigenous Lactobacilli; Salmonella typhimurium

\section{INTRODUCTION}

Lactobacillus is a probiotic bacterium commonly used to alleviate diarrhea and improve nutrient efficiency, promote growth, and enhance the carcass quality of pigs (Hou et al., 2015; Sayan et al., 2018). Another benefit is that Lactobacillus can incapacitate enteric pathogens (Fijan, 2018). A few studies have concerned swine Lactobacilli and the rising trend toward probiotic usage in the pig production industry. The application of Lactobacillus probiotics in livestock production is an alternative to medicine utilization for disease prevention and growth promotion (Hou et al., 2015; Śliżewska et al., 2021). Since livestock is the primary protein source for humans, Lactobacillus probiotics are attractive options concerning the risk minimization of antimicrobialresistant bacteria, drug residues, and pathogen-free foods. However, not all probiotic products are equally effective (Sniffen et al., 2018). The issue is whether non-host-specific probiotics exert maximum beneficial effects; for example, a non-swine origin of probiotic strains may pose difficulties in intestinal colonization and competition with normal flora (Fijan, 2014).

Most commercial Lactobacillus probiotic seed strains are obtained from humans, foods, and plants. Furthermore, the species-specific characteristics of Lactobacilli, particularly for local pigs, are still not well understood, especially in Thailand. Orally administered probiotics should be able to survive the harsh gastrointestinal environment, which includes such components as gastric acid and bile salt; compete with other intestinal residential floras; settle well in the gastrointestinal tract; maintain sufficient numbers; confer positive health effects; and inactivate pathogens (Fenster et al., 2019). In addition to viability, a safety precaution to be considered when using Lactobacillus is that probiotic seeds must not be antibiotic-resistant (Hou et al., 2015). In the present study, indigenous Lactobacillus strains are promising for attaining probiotic effects because they should adapt well in pigs, fit ecological niches, and reduce the risk of exotic bacteria importation. Therefore, studying the probiotic characteristics of Lactobacilli 
isolated from organic pigs is the right foundation for sustainable swine production in northeastern Thailand. This study aimed to evaluate the in vitro probiotic properties of Lactobacillus spp. isolated from the feces of antibiotic-free organic pigs.

\section{MATERIALS AND METHODS}

\section{Standard Bacteria}

Standard bacteria included in the study were Salmonella typhimurium ATCC 13311 (S. typhimurium) and Escherichia coli ATCC 25922 (E. coli) for antagonistic assay and quality control of the susceptibility test, Enterococcus faecalis ATCC 7212 (E. faecalis) for quality control of susceptibility test and bile salt quality control, and Staphylococcus aureus ATCC 25923 (S. aureus) for quality control of the susceptibility test. All standard strains were obtained from the American Type Culture Collection (ATCC).

\section{Lactobacillus Strains}

Lactobacilli were selected from our laboratory collection, with isolates derived from 18 different healthy organic pigs raised under a strict "permaculture farm practice" in Khon Kaen Province, Thailand. Since its establishment 10 years ago, the farm has avoided using any medicines, chemical substances, or vaccines. The farm produces and formulates its feed. Eighteen isolates of the three following species of Lactobacillus have been used in this study: L. mucosae $(\mathrm{n}=10)$, L. reuteri $(\mathrm{n}=7)$, and L. plantarum $(\mathrm{n}=1)$. Names and details of each strain are shown in Table 1. The 18 Lactobacillus cultures were stored at $-20{ }^{\circ} \mathrm{C}$ in De Man-Rogosa-Sharpe broth (MRS, Hi-Media Pvt. Ltd., India) supplemented with horse serum (20\% v/v) and L-cysteine (0.05\% w/v; No. 30089 BioUltra, Sigma-Aldrich (Thailand) Co. Ltd.).

\section{Preparation of Stock Bacteria}

The frozen stored Lactobacillus isolates were cultivated on MRSc agar (MRS broth supplemented with $0.05 \% \mathrm{w} / \mathrm{v}$ of L-cysteine, and $1.5 \% \mathrm{w} / \mathrm{v}$ of bacteriological agar (Hi-Media Pvt. Ltd., India), pH 6.5, and incubated under an anaerobic atmosphere $(17 \%$ CO2, $80 \%$ N2, and $3 \% \mathrm{H} 2$ ) for $48 \mathrm{~h}$ at $37{ }^{\circ} \mathrm{C}$. Fresh and active-grown Lactobacillus colonies were transferred into MRSc broth, $\mathrm{pH}$ 6.5. Following this, the tubes were incubated at 37 ${ }^{\circ} \mathrm{C}$ for $24 \mathrm{~h}$. Before the assays, this Lactobacillus stock was diluted in phosphate-buffered saline supplemented with $0.05 \%$ L-cysteine (PBSc) to prepare the desired concentrations (MacFarland No. 1 to 5) for specific assays. Frozen stored S. typhimurium, E. coli, and E. faecalis were cultured on nutrient agar (Oxoid, United Kingdom) incubated at $37{ }^{\circ} \mathrm{C}$ overnight. Frozen stored S. aureus was cultured on $5 \%$ bovine blood agar (Columbia agar, Oxoid) incubated at $37^{\circ} \mathrm{C}$ overnight. Fresh stock of these reference bacteria was prepared by transferring colonies from nutrient agar grown overnight into brain heart infusion broth (BHI, Hi-Media Pvt. Ltd.) and incubated at $37{ }^{\circ} \mathrm{C}$ for $18-24 \mathrm{~h}$. Before use, the stocks were diluted with PBSc to equal MacFarland No. 1 to 5 as specific concentrations for the assays.

\section{Pepsin Tolerance}

One hundred microliters of Lactobacillus bacterial solution (1.0 MacFarland concentration) was transferred into $1 \mathrm{~mL}$ of $0.3 \%$ pepsin (PanReac-AppliChem, $\mathrm{GmbH}$, Germany) in PBSc, adjusted to $\mathrm{pH} \mathrm{3}$, and $1 \mathrm{~mL}$ PBSc (control) tubes, incubated at $37{ }^{\circ} \mathrm{C}$ for $2 \mathrm{~h}$ (Arboleya et

Table 1. Lactobacillus spp. isolates used in the study

\begin{tabular}{|c|c|c|c|c|}
\hline No. & Isolate & Lactobacillus spp. & Source (pig) & NCBI accession numbers \\
\hline 1 & OP1 & Lactobacillus reuteri & Sow & MZ382874 \\
\hline 2 & OP2 & Lactobacillus mucosae & Piglet & MZ382875 \\
\hline 3 & OP3 & Lactobacillus mucosae & Sow & MZ382876 \\
\hline 4 & OP4 & Lactobacillus mucosae & Finishing & MZ382877 \\
\hline 5 & OP5 & Lactobacillus mucosae & Finishing & MZ382878 \\
\hline 6 & OP6 & Lactobacillus mucosae & Finishing & MZ382879 \\
\hline 7 & OP7 & Lactobacillus plantarum & Finishing & MZ382880 \\
\hline 8 & OP8 & Lactobacillus mucosae & Finishing & MZ382881 \\
\hline 9 & OP9 & Lactobacillus reuteri & Finishing & MZ382882 \\
\hline 10 & OP10 & Lactobacillus reuteri & Finishing & MZ382883 \\
\hline 11 & OP11 & Lactobacillus mucosae & Piglet & MZ382884 \\
\hline 12 & OP12 & Lactobacillus mucosae & Piglet & MZ382885 \\
\hline 13 & OP13 & Lactobacillus mucosae & Finishing & MZ382886 \\
\hline 14 & OP14 & Lactobacillus reuteri & Sow & MZ382887 \\
\hline 15 & OP15 & Lactobacillus reuteri & Sow & MZ382888 \\
\hline 16 & OP16 & Lactobacillus mucosae & Growing & MZ382889 \\
\hline 17 & OP17 & Lactobacillus reuteri & Growing & MZ382890 \\
\hline 18 & OP18 & Lactobacillus reuteri & Growing & MZ382891 \\
\hline
\end{tabular}

Note: All strains were identified by genomic sequencing of 16S rRNA using universal primers; 27F: 5' - AGAGTTTGATCCTGGCTCAG - 3' and 1492R: 5' -GGTTACCTTGTTAGGACTT- 3' and compared to the sequence database in GenBank (http://www.ncbi.nim.nih.gov) by BLAST program. $\mathrm{OP}=$ organic pigs. 
al., 2011). Surviving Lactobacilli from the tubes were checked by inoculating the loop of the bacterial solution on MRSc agar plates and incubated anaerobically at 37 ${ }^{\circ} \mathrm{C}$ for $24-48 \mathrm{~h}$, observed for Lactobacillus characteristic colonies. Growth ability was reported by grading the colonies with one to three plus signs compared with control. An invisible colony on agar plates was read as no growth.

\section{Bile Salt Tolerance}

The endurance test to $2 \%$ bile salt was similar to the earlier method (Awasti et al., 2016) with a slight change. Fifty microliters (1.0 MacFarland concentration) each of Lactobacillus and E. faecalis (Standard bile resistance control) suspension was inoculated into $2 \mathrm{~mL}$ MRSc broth supplemented with $150 \mu \mathrm{L}$ of $29 \%$ stock solution of bile salt (Hi-Media Pvt. Ltd.) to obtain a $2 \%$ final concentration of bile salt in the MRSc broth. At the same time, control tubes were prepared by transferring $50 \mu \mathrm{L}$ of the same stock Lactobacillus and E. faecalis into $2 \mathrm{~mL}$ MRSc broth without bile salt. After $3 \mathrm{~h}$ of incubation at $37^{\circ} \mathrm{C}$, a loop of the bacterial solution was plated on MRSc or nutrient agars (E. faecalis) and incubated anaerobically at $37{ }^{\circ} \mathrm{C}$ for $24-48 \mathrm{~h}$. Then, the plates were observed for Lactobacillus or E. faecalis characteristic colonies. Qualitative survived Lactobacillus was graded the colonies compared with control as an aforesaid method.

\section{Temperature Tolerance}

Temperature and duration sets were for survival ability of Lactobacillus storage and shelf life $\left(37^{\circ} \mathrm{C}, 4{ }^{\circ} \mathrm{C}\right)$ and after manufacturing or packaging of probiotic seeds $\left(50{ }^{\circ} \mathrm{C}\right)$. One hundred microliters of overnight grown Lactobacillus (1.0 MacFarland concentration) were inoculated into a $4 \mathrm{~mL}$ MRSc broth tube and incubated anaerobically at $37{ }^{\circ} \mathrm{C}$ for 5 and 7 days; $50{ }^{\circ} \mathrm{C}$ for 1,6 , and $24 \mathrm{~h}$; and $4{ }^{\circ} \mathrm{C}$ for $1,6,10$, and 14 days. For each specific time point, bacterial enumeration proceeded by plate count agar. Serial tenfold dilutions of the bacterial solutions were prepared and spread $(100 \mu \mathrm{L})$ on MRSc agar plates. After anaerobic incubation at $37{ }^{\circ} \mathrm{C}$ for $24-$ $48 \mathrm{~h}$, Lactobacillus colonies were counted and calculated following the dilutions and expressed as colony-forming units per milliliter $(\mathrm{CFU} / \mathrm{mL})$. Except for the solution treated at $50{ }^{\circ} \mathrm{C}$ for $24 \mathrm{~h}$, these were evaluated as alive or inactivated.

\section{Aerotolerance}

One hundred microliters of overnight grown Lactobacillus suspension (1.0 MacFarland concentration) were transferred into $4 \mathrm{~mL} \mathrm{MRSc}$ broth, and one mixture in a loose-cap tube and another one in a tightened screw-cap tube (control) were prepared. The loose-cap tubes were on a rack intact, and the tightened screw-cap tubes were in an anaerobic chamber for aerobic and anaerobic incubation, respectively. After incubation at $37{ }^{\circ} \mathrm{C}$ overnight, one solution loop was streak on MRSc agar, incubated anaerobically at $37{ }^{\circ} \mathrm{C}$ for $24-48$ h. Growth ability was reported by grading the colonies with one to three plus signs compared with control as the aforesaid method.

\section{Antimicrobial Susceptibility Test}

The in vitro antibiotic susceptibility test was employed with the disk diffusion method following the guidelines of the Clinical and Laboratory Standards Institute (CLSI, 2018). Twelve antibiotics (Oxoid) were included as follows: gentamicin $10 \mu \mathrm{g}$, streptomycin $10 \mu \mathrm{g}$, cefotaxime $30 \mu \mathrm{g}$, norfloxacin $10 \mu \mathrm{g}$, amoxicillin clavulanate $30 \mu \mathrm{g}$, clindamycin $2 \mu \mathrm{g}$, ampicillin $10 \mu \mathrm{g}$, sulfamethoxazole-trimethoprim $25 \mu \mathrm{g}$, enrofloxacin 5 $\mu \mathrm{g}$, tetracycline $30 \mu \mathrm{g}$, imipenem $10 \mu \mathrm{g}$, and vancomycin $30 \mu \mathrm{g}$. The standard quality control bacteria were also included. Susceptibility interpretation followed the standard breakpoints of E. faecalis and S. aureus recommended by the CLSI-M100-S28 guideline (CLSI, 2018).

\section{Antagonistic Test}

The antagonist effect of Lactobacillus on the growth of S. typhimurium ATCC 13311 and E. coli ATCC 25922 was tested by co-culture in MRSc broth (Ahn et al., 2002). Single cultures of Lactobacillus, S. typhimurium, or $E$. coli were prepared along the co-culture inoculums. In brief, $50 \mu \mathrm{L}$ (1.0 MacFarland concentration) of each bacterial solution was inoculated into $2 \mathrm{~mL}$ MRSc broth tubes. The co-culture tubes composed of $50 \mu \mathrm{L}$ of either S. typhimurium or E. coli and $50 \mu \mathrm{L}$ of each Lactobacillus strain were transferred into a $2 \mathrm{~mL}$ MRSc broth tube. The preparation obtained was three different sets of bacteria-namely, Lactobacillus only (Lacto), S. typhimurium only (Sal), and Lactobacillus plus S. typhimurium $(\mathrm{L}+\mathrm{S})$. Similar processes were done for $E$. coli, with sets of Lactobacillus only (Lacto), E. coli only (Ecoli), and Lactobacillus plus E. coli ( $\mathrm{L}+\mathrm{E})$. The single or combination inoculums were incubated anaerobically at $37{ }^{\circ} \mathrm{C}$ for 24 $\mathrm{h}$. Live bacteria from the co-culture broth were then enumerated using MRSc agar (adjusted to pH 5) selectively for Lactobacillus and MacConkey agar (Oxoid) for E. coli and S. typhimurium.

\section{RESULTS}

\section{Tolerance to Bile Salt, Pepsin, $50^{\circ} \mathrm{C}$ for $24 \mathrm{~h}$, and the Presence of Oxygen}

A qualitative assessment of Lactobacillus was determined for the tolerance to bile salt, pepsin, $50{ }^{\circ} \mathrm{C}$ for 24 $h$, and the presence of oxygen. Ten strains isolated from organic pigs or OP (OP1, OP2, OP3, OP7, OP9, OP10, OP11, OP15, OP17, and OP18) were qualitatively alive after $3 \mathrm{~h}$ of exposure to $2 \%$ bile salt; OP4, OP5, OP6, OP12, and OP16 did not survive after bile salt exposure. The activity of OP8, OP13, and OP14 diminished noticeably when incubated with $2 \%$ bile salt for $3 \mathrm{~h}$ (Table 2). All the strains endured $0.3 \%$ pepsin for $2 \mathrm{~h}$, and no obvious reduction in the activity was observed (Table 2). Eight strains-OP1, OP2, OP3, OP8, OP9, OP10, OP11, and OP15-stayed alive after being incubated at $50{ }^{\circ} \mathrm{C}$ for 24 h. However, seven strains-OP5, OP7, OP12, 
OP13, OP16, OP17, and OP18-were completely inactivated, and three strains-OP4, OP6, and OP14-were barely alive (Table 2).

\section{Temperature Tolerance}

From the results of Lactobacillus enumeration, there was no apparent difference between day zero (D0, the starting point) and incubation at $50{ }^{\circ} \mathrm{C}$ for $1 \mathrm{~h}$ (range: -0.43 to $0.44 \log _{10} \mathrm{CFU} / \mathrm{mL}$ ). In comparison, viable bacteria for all the isolates decreased (from D0) from 1.2 to $4.93 \log _{10} \mathrm{CFU} / \mathrm{mL}$ after incubation at $50{ }^{\circ} \mathrm{C}$ for 6 h (Table 3). Five isolates-OP1, OP9, OP14, OP15, and OP18-remained stable after incubation at $50{ }^{\circ} \mathrm{C}$ for 6 h (decreased less than $2 \log _{10} \mathrm{CFU} / \mathrm{mL}$ ). However, OP7 and OP16 were the most affected by high temperatures (decreased more than $4 \log _{10} \mathrm{CFU} / \mathrm{mL}$; Table. 3). There were two isolates, OP1 and OP2, that performed well after being placed at $37{ }^{\circ} \mathrm{C}$ for 5 days (decreased 1.45 and $2.38 \log _{10} \mathrm{CFU} / \mathrm{mL}$, respectively). With this condition, OP6, OP7, OP16, and OP17 decreased more than 4 $\log _{10} \mathrm{CFU} / \mathrm{mL}$; OP14 decreased $2.74 \log _{10} \mathrm{CFU} / \mathrm{mL}$, and the other isolates decreased more than $3 \log _{10} \mathrm{CFU} / \mathrm{mL}$ (Table 3). After 7 days at $37^{\circ} \mathrm{C}, \mathrm{OP} 2, \mathrm{OP6}$, OP16, and

Table 2. Qualitative assessment of Lactobacillus spp. isolates regarding tolerance to bile salt, pepsin, $50{ }^{\circ} \mathrm{C}$ for $24 \mathrm{~h}$, and the presence of oxygen

\begin{tabular}{|c|c|c|c|c|c|}
\hline Isolate & $2 \%$ bile salt & $0.3 \%$ pepsin & PBSc (control) & $50^{\circ} \mathrm{C} 24 \mathrm{~h}$ & Aerotolerance \\
\hline OP1 & +++ & +++ & +++ & +++ & +++ \\
\hline OP2 & +++ & +++ & +++ & +++ & +++ \\
\hline OP3 & +++ & +++ & +++ & +++ & +++ \\
\hline OP4 & - & +++ & +++ & + & +++ \\
\hline OP5 & - & +++ & +++ & - & +++ \\
\hline OP6 & - & +++ & +++ & + & +++ \\
\hline OP7 & +++ & +++ & +++ & - & +++ \\
\hline OP8 & + & +++ & +++ & +++ & +++ \\
\hline OP9 & +++ & +++ & +++ & +++ & +++ \\
\hline OP10 & +++ & +++ & +++ & +++ & +++ \\
\hline OP11 & +++ & +++ & +++ & +++ & +++ \\
\hline OP12 & - & +++ & +++ & - & +++ \\
\hline OP13 & + & +++ & +++ & - & +++ \\
\hline OP14 & + & +++ & +++ & + & +++ \\
\hline OP15 & +++ & +++ & +++ & +++ & +++ \\
\hline OP16 & - & +++ & +++ & - & +++ \\
\hline OP17 & +++ & +++ & +++ & - & +++ \\
\hline OP18 & +++ & +++ & +++ & - & +++ \\
\hline
\end{tabular}

Note: +++ = grow well; += poor growth; - = no growth; OP= organic pigs; PBSc= phosphate-buffered saline supplemented with $0.05 \%$ L-cysteine.

Table 3. Enumeration of Lactobacillus spp. isolates after incubation to various temperatures (log $\left.\mathrm{CFU}_{10} / \mathrm{mL}\right)$

\begin{tabular}{|c|c|c|c|c|c|c|c|c|c|}
\hline \multirow{2}{*}{ Isolate } & \multirow{2}{*}{ D0 } & \multicolumn{2}{|c|}{$50^{\circ} \mathrm{C}$} & \multicolumn{4}{|c|}{$4^{\circ} \mathrm{C}$} & \multicolumn{2}{|c|}{$37^{\circ} \mathrm{C}$} \\
\hline & & $1 \mathrm{~h}$ & $6 \mathrm{~h}$ & D1 & D6 & D10 & D14 & D5 & D7 \\
\hline OP1 & 8.22 & 8.32 & 7.00 & 8.48 & 8.61 & 7.98 & 9.38 & 6.77 & 5.65 \\
\hline OP2 & 9.51 & 9.36 & 6.60 & 10.04 & 9.54 & 9.27 & 9.26 & 7.13 & 3.95 \\
\hline OP3 & 9.16 & 8.89 & 7.26 & 8.96 & 8.54 & 8.55 & 9.26 & 6.07 & 5.88 \\
\hline OP4 & 9.51 & 9.30 & 6.08 & 9.44 & 9.51 & 9.20 & 9.19 & 5.74 & 4.90 \\
\hline OP5 & 9.52 & 9.36 & 6.91 & 9.43 & 9.12 & 9.45 & 8.99 & 5.83 & 5.26 \\
\hline OP6 & 10.08 & 9.86 & 7.61 & 10.30 & 9.36 & 9.93 & 9.56 & 4.40 & 3.53 \\
\hline OP7 & 10.43 & 10.31 & 5.72 & 10.08 & 10.33 & 10.5 & 10.49 & 4.88 & NG \\
\hline OP8 & 10.34 & 9.94 & 7.05 & 9.53 & 9.70 & 9.55 & 9.25 & 6.53 & 5.99 \\
\hline OP9 & 10.04 & 10.47 & 8.72 & 9.76 & 9.73 & 9.55 & 9.60 & 7.01 & 5.24 \\
\hline OP10 & 9.72 & 9.75 & 7.03 & 9.42 & 9.88 & 9.77 & 9.43 & 6.42 & 5.08 \\
\hline OP11 & 9.23 & 9.10 & 6.97 & 9.12 & 9.38 & 9.53 & 8.66 & 5.89 & 5.07 \\
\hline OP12 & 10.04 & 10.38 & 7.63 & 9.54 & 9.48 & 9.13 & 8.39 & 6.40 & 5.63 \\
\hline OP13 & 9.44 & 9.62 & 7.11 & 9.35 & 9.49 & 9.17 & 8.88 & 6.13 & 5.51 \\
\hline OP14 & 9.16 & 10.19 & 7.96 & 9.05 & 9.12 & 9.08 & 8.72 & 6.42 & 5.98 \\
\hline OP15 & 10.02 & 9.67 & 8.70 & 9.46 & 9.31 & 9.29 & 8.65 & 6.71 & 6.99 \\
\hline OP16 & 10.39 & 9.95 & 5.46 & 9.81 & 9.45 & 9.32 & 8.86 & 5.66 & 1.60 \\
\hline OP17 & 9.67 & 9.68 & 6.64 & 9.51 & 9.57 & 8.86 & 8.87 & 3.74 & 3.78 \\
\hline OP18 & 9.42 & 9.52 & 7.43 & 9.94 & 7.79 & 7.00 & 7.95 & 5.62 & 5.35 \\
\hline
\end{tabular}

Note: NG= no growth; D0= Day0; D1= Day1; D5= Day 5; D6= Day6; D7= Day7; D10= Day10; D14= Day 14; OP= organic pigs. 
OP17 decreased more than $5 \log _{10} \mathrm{CFU} / \mathrm{mL}$ and OP7 was completely lost all activity (Table 3 ). For the refrigerated storage temperature of $4{ }^{\circ} \mathrm{C}$, the numbers for live bacteria fluctuated between 1.16 and $2.42 \log _{10} \mathrm{CFU} / \mathrm{mL}$ for all the isolates as counted on D1, D6, D10, and D14 (Table $3)$.

\section{Antagonistic Activity}

Whether a single Lactobacillus culture was used or a combination with either S. typhimurium ATCC 13311 or E. coli ATCC 25922, the numbers of Lactobacillus were almost the same (Figure 1 and 2). In contrast, the numbers of both pathogens distinctly reduced in the co-culture tubes until they were unculturable. Eleven strains of all tested Lactobacilli-OP1-4, OP7, OP10, OP12, OP14, and OP16-18-completely inhibited S. typhimurium and E. coli (Figure 1 and 2). The OP9 inactivated S. typhimurium completely but only caused a reduction in the amount of E. coli (31.2\% reduction). Four strains-OP5, OP6, OP8, and OP15-perfectly antagonized E. coli growth but only diminished numbers of $S$. typhimurium, with $71 \%$, $44 \%, 27 \%$, and $45.6 \%$ reduction rates, respectively. Two isolates, OP11 and OP13, reduced the number of $S$. $t y$ phimurium by $88.8 \%$ and $34.9 \%$ and reduced the number of E. coli by $27.8 \%$ and $48.2 \%$, respectively.

\section{Antimicrobial Susceptibility}

All Lactobacilli were completely susceptible to amoxicillin-clavulanate, ampicillin, and imipenem. Varying susceptibility (range: 39\%-56\%) was found among the 18 Lactobacillus isolates to the following drugs: gentamicin, streptomycin, cefotaxime, and tetracycline (Figure 3). Most isolates were sensitive to clindamycin $(72 \%)$, gentamicin $(56 \%)$, and tetracycline (50\%). A small proportion of isolates were sensitive to cefotaxime (39\%) and streptomycin (39\%). Inhibition zones that fell into intermediate susceptibility were found in cefotaxime $(17 \%)$, clindamycin $(11 \%)$, tetra-

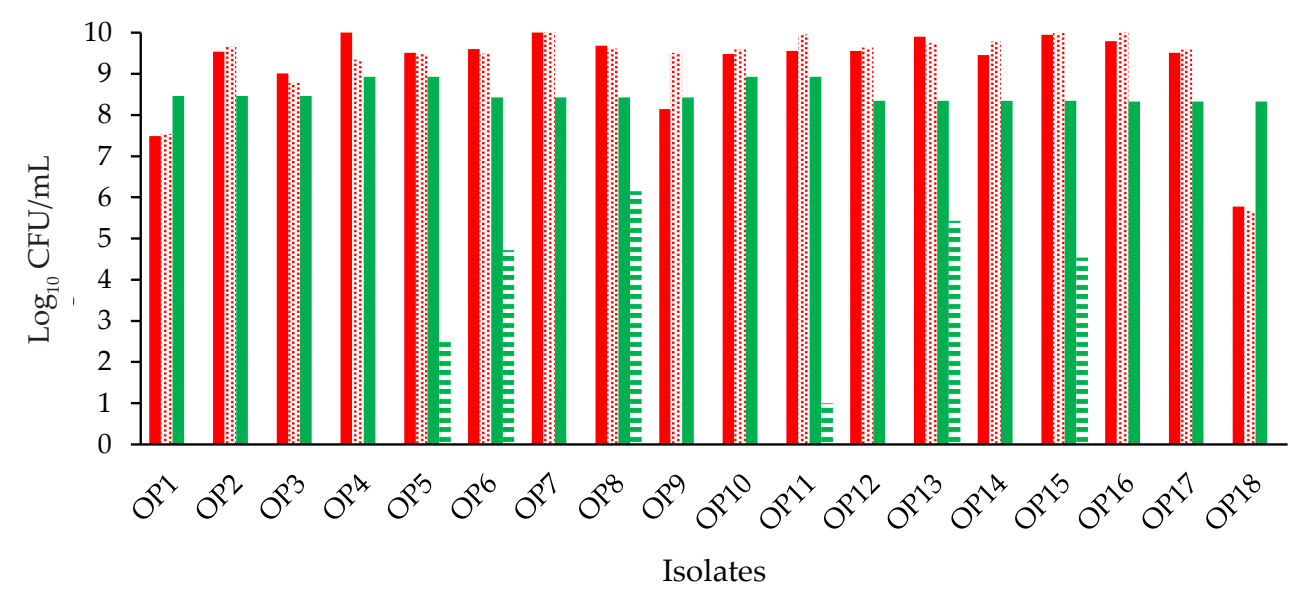

Figure 1. Bacterial numbers determined by the viable plate count agar method after single culture (Lacto) or co-culture (L+S) between Lactobacillus spp. isolates and Salmonella typhimurium ATCC 13311.

Note: $0=$ no growth, Lacto $(\square)=$ Lactobacillus, Sal $(\square)=$ Salmonella, Lacto $(\mathrm{L}+\mathrm{S})(\mathrm{n})=$ Lactobacillus in the co-cultured tube, $\mathrm{Sal}(\mathrm{L}+\mathrm{S})(=)=$ Salmonella in the co-cultured tube; $\mathrm{OP}=$ organic pigs.

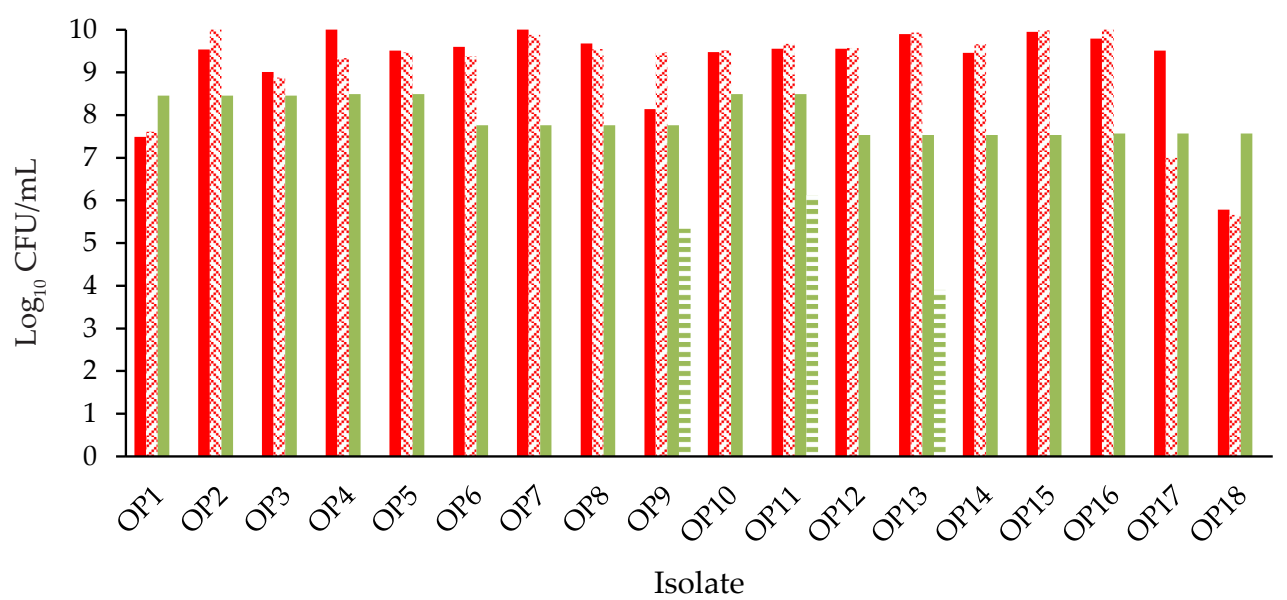

Figure 2. Bacterial number determined by the viable plate count agar method after single culture (Lacto) or co-culture (L+E) between Lactobacillus spp. isolates and Eschericia coli ATCC 25922.

Note: $0=$ no growth, Lacto $(\square)=$ Lactobacillus, Ecoli $(\square)=$ Eschericia coli, Lacto $(\mathrm{L}+\mathrm{E})(\mathrm{N})=$ Lactobacillus in the cocultured tube, Ecoli $(\mathrm{L}+\mathrm{E})(=)=$ Eschericia coli in the co-cultured tube; $\mathrm{OP}=$ organic pigs. 


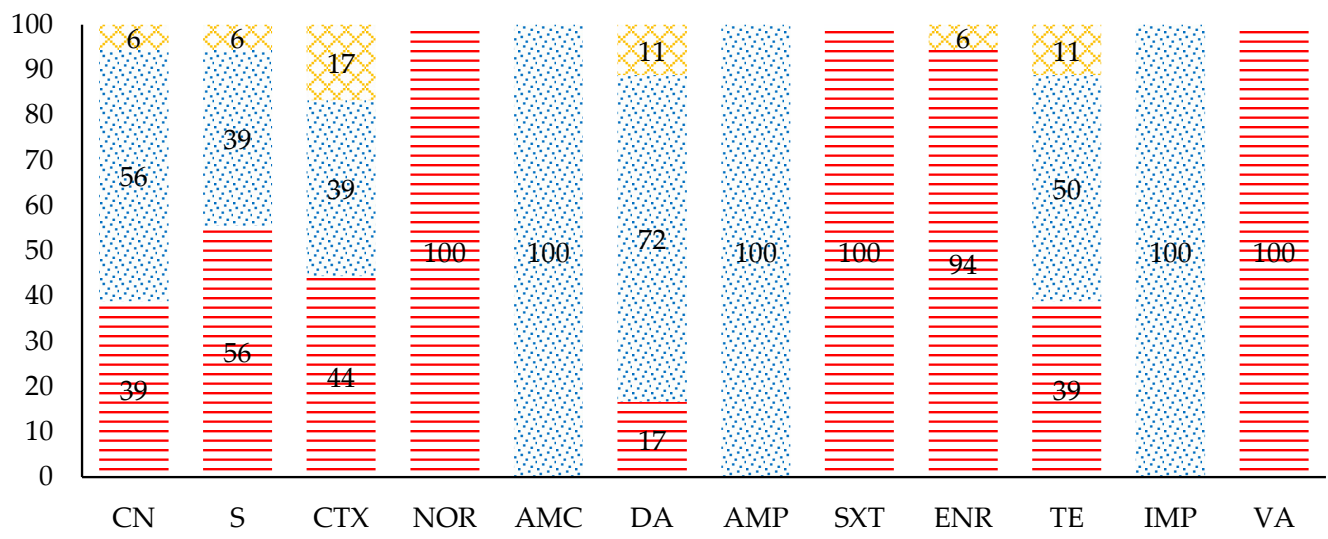

Figure 3. Antibiogram results of 18 Lactobacillus spp. isolates against 12 drugs in the study, $\equiv=\%$ Resistance, $=\%$ Susceptible, $\&=\%$ Intermediate.

Note: $\mathrm{CN}=$ Gentamicin $(10 \mu \mathrm{g}), \mathrm{S}=$ Streptomycin $(10 \mu \mathrm{g}), \mathrm{CTX}=$ Cefotaxime $(30 \mu \mathrm{g}), \mathrm{NOR}=$ Norfloxacin $(10 \mu \mathrm{g}), \mathrm{AMC}=$ Amoxicillin-clavulanate $(30 \mu \mathrm{g}), \mathrm{DA}=$ Clindamycin $(2 \mu \mathrm{g}), \mathrm{AMP}=$ Ampicillin $(10 \mu \mathrm{g})$, $\mathrm{SXT}=$ Sulfamethoxazole -trimethoprim $(25 \mu \mathrm{g}), \mathrm{ENR}=$ Enrofloxacin $(5 \mu \mathrm{g}), \mathrm{TE}=$ Tetracycline $(30 \mu \mathrm{g}), \mathrm{IPM}=$ Imipenem $(10 \mu \mathrm{g}), \mathrm{VA}=$ Vancomycin $(30 \mu \mathrm{g})$.

cycline $(11 \%)$, gentamicin $(6 \%)$, and enrofloxacin $(6 \%$; Figure 3). All strains showed $100 \%$ resistance against norfloxacin, sulfamethoxazole-trimethoprim, vancomycin resistance, and $94 \%$ against enrofloxacin. None of the Lactobacilli tested was susceptible to enrofloxacin, but some strains had intermediate susceptibility to this drug.

Variable resistance was detected for streptomycin $(56 \%)$, cefotaxime $(44 \%)$, gentamicin $(39 \%)$, tetracycline (39\%), and clindamycin (17\%). The result indicated drug resistance if equal to or more than half of the isolates were resistant. Hence, streptomycin $(56 \%)$ was associated with moderate resistance. Half the proportions of Lactobacilli were somewhat sensitive/resistant to cefotaxime $(39 / 44 \%)$, streptomycin $(39 / 56 \%)$, and tetracycline (50/39\%). Except for OP7, OP9, and OP18, the other Lactobacillus isolates were sensitive to clindamycin (Table 3). More than half of the isolates exhibited streptomycin resistance, but OP1, OP2, OP3, OP13, OP14, OP17, and OP18 were sensitive to this drug.

\section{DISCUSSION}

Lactobacilli can be found along the length of the gastrointestinal tract and are abundant in the large intestine (Wylensek et al., 2020), which is the targeted residency of exogenous probiotic administration. Furthermore, Lactobacillus probiotics utilized in pigs are mostly administered in feed additives (Alayande et al., 2020). Our results indicated that all Lactobacillus isolates tolerated gastrointestinal conditions. Survival of Lactobacillus may be affected by the duration of pepsin and bile salt exposure as well. The duration of $2-3 \mathrm{~h}$ conducted in our study was compatible with the gastric transit time of the pig (Henze et al., 2021). It may be that certain amounts of the bacteria had lost activity, but our result identified them simply as qualitatively alive. The pepsin used in our study was derived from swine, which affirms that pigs' Lactobacilli can resist the gastric environment. For the bile salt test, our result corroborates another study finding that Lactobacillus of pig origin remained alive after exposure to $2 \%$ bile salt (Betancur et al., 2020). Bile salt is present in the small and large intestines; this is why it is assumed that Lactobacilli obtained from pig feces can endure the bile salt test (Ruiz et al., 2013). Ten isolates evaluated in this study are promising for oral probiotic preparations. Although the study did not evaluate the exact numbers of Lactobacilli remaining after bile salt and pepsin exposure, live bacteria can proliferate in the intestine once residing there. Although the intestinal adherence ability of Lactobacilli was not investigated in this study, all strains were procured from the rectums of healthy pigs, which ensured their natural habitat.

For manufacturing criteria, probiotic starter culture must maintain viability in sufficient numbers after undergoing probiotic production steps and storage temperatures. Oxygen exposure during handling is another issue involved in anaerobic bacteria manipulation. The species of the genus Lactobacillus have been classified as oxygen-tolerant anaerobes (Zotta et al., 2017). The results of our study indicated that all isolates could grow in the microaerophilic atmosphere. An additional property of probiotics is that they must tolerate high temperatures. All Lactobacillus strains tested could resist $50{ }^{\circ} \mathrm{C}$ for $6 \mathrm{~h}$, although they were slightly reduced in number. Generally, Lactobacilli were not able to grow under hightemperature conditions (Praepanitchai et al., 2019). As a result, most isolates diminished in terms of their viable numbers when incubated at $50{ }^{\circ} \mathrm{C}$ for $24 \mathrm{~h}$ or $37{ }^{\circ} \mathrm{C}$ for 7 days, although some isolates still had growth activity. The high-temperature resistance of the pig's Lactobacilli could be explained by the pigs' high body temperature. The normal rectal temperature of pigs is $38.5{ }^{\circ} \mathrm{C}$ to $39.5{ }^{\circ} \mathrm{C}$ (Zhang et al., 2019), and the temperature in the abdominal cavity (where the intestine is located) can be much higher than this. For the storage temperature, there was no dispute that most of our Lactobacilli could maintain good activity at $4{ }^{\circ} \mathrm{C}$ for up to 14 days, and this result agreed well with another study (Watkins et 
al., 2018). Nevertheless, keeping them at $4{ }^{\circ} \mathrm{C}$ for longer than 14 days was not attempted in our study.

Beyond good attributes concerning the handling, safety issues of probiotic seeds, such as drug resistance, must be cautiously considered (Anisimova \& Yarullina, 2020). The present study established the antimicrobial susceptibility standard of Lactobacillus from antibioticfree pigs raised on a 10-year-old farm that had never used any chemicals or medicines. As a matter of fact, the true origin of Lactobacillus strains was unknown. Although procured from pigs on the same farm, various reactions of antimicrobial susceptibility were found among 18 Lactobacillus isolates. The previous study analyzed six standard reference Lactobacillus strains that yield variable activity against antimicrobials, similar to our results (Sharma et al., 2017). The variable antibiogram patterns could be due to the species and origin of Lactobacillus. According to a study that investigated $L$. salivarius and L. mucosae derived from the same wild boar, these Lactobacilli exhibited different drug susceptibility patterns (Fukuda et al., 2020). In our study, all Lactobacillus isolates were completely susceptible to ampicillin, amoxicillin-clavulanate, and imipenem, as expected for Gram-positive bacteria. Most strains were sensitive to clindamycin. Moderate sensitivity of a few Lactobacilli toward clindamycin was also indicated in a previous study (Schmitt et al., 2018). Our isolates exhibited variable sensitivity/resistance to gentamicin and streptomycin, while another study reported that their isolates were highly sensitive to these two antibiotics (Georgieva et al., 2015). Resistance phenotype to aminoglycosides of Lactobacillus has been addressed as intrinsic (Hummel et al., 2007). However, another study demonstrated aminoglycosides' resistance genes in Lactobacilli procured from chickens (Dec et al., 2017).

Small numbers of our isolates were resistant to tetracycline, but more than half of these Lactobacilli were susceptible to this drug. It can be concluded that our isolates were tetracycline susceptible. Although 100\% tetracycline resistance was reported by other researchers (Anisimova \& Yarullina, 2018), Lactobacilli were found to be completely susceptible to tetracycline in a different study (Jomehzadeh et al., 2020). Extensive exploration of tetracycline resistance using 128 strains of the L. buchneri group, the research team concluded that the tetracycline resistance was intrinsic (Feichtinger et al., 2016). They found that $96.9 \%$ of the strains could be categorized as tetracycline-resistant, but none of the Lactobacilli carried tetracycline resistance genes (Feichtinger et al., 2016). Less than half the 18 isolates fell into either sensitivity or resistance against cefotaxime (with almost equal proportions). It can be inferred that our Lactobacilli were somewhat sensitive to this drug, which is similar to the findings of a previous study in that Lactobacillus strains were completely sensitive to $\beta$-lactam antibiotics but moderately sensitive to cefotaxime (Sharma et al., 2017).

All Lactobacilli in our study were completely resistant to norfloxacin, sulfamethoxazole-trimethoprim, and vancomycin, and $94 \%$ of the isolates were resistant to enrofloxacin. None of the Lactobacilli tested were susceptible to enrofloxacin; this was considered to indicate enrofloxacin resistance. High resistance toward glycopeptides (vancomycin, teicoplanin) and quinolones (ciprofloxacin, ofloxacin) have been demonstrated in reference Lactobacillus commercial strains (Sharma et al., 2017). It has been indicated that Lactobacilli possess intrinsic resistance to these two antibiotic classes (Casado Muñoz et al., 2014). Natural resistance to vancomycin is due to Lactobacillus having peptidoglycan precursors terminating in D-alanyl-D-lactate. Vancomycin binds relatively poorly to this peptidoglycan ending, whereas it binds with high affinity to peptidoglycan ending in D-alanyl-D-alanine, causing natural vancomycin resistance of Lactobacillus (Zhang et al., 2018). Insensitivity to enrofloxacin was also detected in Lactobacillus isolated from weaned pigs (Zou et al., 2017). Nevertheless, no mutations in the quinolone resistance determining regions of the genes encoding GyrA and ParC of Lactobacilli were found, indicating an intrinsic resistance (Casado Muñoz et al., 2014). Intrinsic resistance is usually nontransferable, and hence, poses no risk for using Lactobacillus as probiotics. Further screenings and indepth characterization of the resistant determinants are required to use Lactobacillus starter culture safely.

Some beneficial bacteria, including Lactobacillus, can produce substances to prevent or inhibit harmful bacteria, and their metabolites contribute to nutritional efficiency and immunological modification of the hosts (Novik \& Savich, 2020). In modern intensive farming systems, high stocking rates predispose pigs to many infectious diseases (Lee et al., 2016). The most costly disease is pre-and post-weaning diarrhea (Breda et al., 2017). The common etiology of this problem relates to Salmonella spp. and E. coli infections (Komatsu et al., 2019). Manipulations of intestinal microbial ecosystems have been attempted to prevent diarrhea, improve health status, and promote growth performance in pigs (Fouhse et al., 2016; Novik \& Savich, 2020). In the present study, autogenous pig Lactobacilli showed good ability to inhibit E. coli and S. typhimurium. The numbers of pathogens were markedly reduced until they were not culturable. Eleven isolates of Lactobacilli completely inactivated both S. typhimurium and E. coli. Notably, some isolates could kill either S. typhimurium or E. coli but not both pathogens. Nevertheless, they could substantially reduce the numbers of culturable $S$. typhimurium and $E$. coli. From this in vitro result, Lactobacilli are promising probiotics to be used for fighting enteric pathogens in swine. It appears that most of our Lactobacilli can combat $E$. coli better than they can combat S. typhimurium. However, this result is preliminary in vitro experiment and cannot elucidate the Lactobacilli's mechanisms. Theoretically, Lactobacillus can produce acids, antimicrobials, and/or other metabolic substances to combat pathogens (Fijan, 2018). In addition, probiotics are acknowledged for being good at completing with pathogens on mucosal adhesion in the intestinal tract (Singh et al., 2017). Further investigation on this attribute is required. 


\section{CONCLUSION}

Four isolates among 18 Lactobacilli-OP1, OP2, $\mathrm{OP} 3$, and OP17-had good in vitro probiotic characteristics. These local Lactobacillus strains are promising commercial probiotics for pigs and are worth further in vivo testing.

\section{CONFLICT OF INTEREST}

We certify that there is no conflict of interest with any financial, personal, or other relationships with other people or organizations related to the materials discussed in the manuscript.

\section{ACKNOWLEDGEMENT}

This work was funded by the Research grant for graduate students of the Faculty of Veterinary Medicine, Khon Kaen University (Project No. 625180005-7/2020).

\section{REFERENCES}

Ahn, Y. T., K. L. Lim, J. C. Ryu, D. K. Kang, J. S. Ham, Y. H. Jang, \& H. U. Kim. 2002. Characterization of Lactobacillus acidophilus isolated from piglets and chicken. Asian-Australas. J. Anim. Sci. 15:1790-1797. https://doi. org/10.5713/ajas.2002.1790

Alayande, K. A., O. A. Aiyegoro, \& C. N. Ateba. 2020. Probiotics in animal husbandry: Applicability and associated risk factors. Sustainability 12:1087. https://doi.org/10.3390/ su12031087

Anisimova, E. \& D. Yarullina. 2018. Characterization of Erythromycin and Tetracycline resistance in Lactobacillus fermentum strains. Int. J. Microbiol. 2018:3912326. https:// doi.org/10.1155/2018/3912326

Anisimova, E. A. \& D. R. Yarullina. 2020. Antibiotic resistance and the mobility of its genetic determinants in Lactobacillus fermentum. Mol. Gen. Microbiol. Virol. 35:202-209. https:// doi.org/10.3103/S0891416820040035

Arboleya, S., P. Ruas-Madiedo, A. Margolles, G. Solís, S. Salminen, G. C. de los Reyes-Gavilán, \& A. Gueimonde. 2011. Characterization and in vitro properties of potentially probiotic Bifidobacterium strains isolated from breast-milk. Int. J. Food Microbiol. 149:28-36. https://doi.org/10.1016/j. ijfoodmicro.2010.10.036

Awasti, N., S. K. Tomar, S. D. Pophaly, P. Poonam, V. K. Lule, T. P. Singh, \& S. Anand. 2016. Probiotic and functional characterization of bifidobacteria of Indian human origin. J. Appl. Microbiol. 120:1021-1032. https://doi.org/10.1111/ jam.13086

Betancur, C., Y. Martínez, G. Tellez-isaias, M. C. Avellaneda, \& B. Velázquez-martí. 2020. In vitro characterization of indigenous probiotic strains isolated from colombian creole pigs. Animals 10:1204. https://doi.org/10.3390/ani10071204

Breda, L.K. Van, O. P. Dhungyel, A. N. Ginn, J. R. Iredell, \& P. Ward. 2017. Pre- and post-weaning scours in southeastern Australia: A survey of 22 commercial pig herds and characterisation of Escherichia coli isolates. PLoS ONE 12:e0172528. https://doi.org/10.1371/journal.pone.0172528

Casado Muñoz, M. del C., N. Benomar, L. L. Lerma, A. Gálvez, \& H. Abriouel. 2014. Antibiotic resistance of Lactobacillus pentosus and Leuconostoc pseudomesenteroides isolated from naturally-fermented Aloreña table olives throughout fermentation process. Int. J. Food Microbiol. 172:110-118. https://doi.org/10.1016/j.ijfoodmicro.2013.11.025
CLSI (Clinical and Laboratory Standards Institute). 2018. Performance Standards for Antimicrobial Susceptibility Testing. 28 $8^{\text {th }}$ Ed. CLSI standard M100. Clinical and Laboratory Standards Institute, Wayne, PA.

Dec, M., R. Urban-Chmiel, D. Stẹpień-Pyśniak, \& A. Wernicki. 2017. Assessment of antibiotic susceptibility in Lactobacillus isolates from chickens. Gut Pathog. 9:54. https://doi. org/10.1186/s13099-017-0203-z

Feichtinger, M., S. Mayrhofer, W. Kneifel, \& K. J. Domig. 2016. Tetracycline resistance patterns of Lactobacillus buchneri group strains. J. Food Prot. 79:1741-1747. https://doi. org/10.4315/0362-028X.JFP-15-577

Fenster, K., B. Freeburg, C. Hollard, C. Wong, R. R. Laursen, \& A. C. Ouwehand. 2019. The production and delivery of probiotics: A review of a practical approach. Microorganisms 7:83. https://doi.org/10.3390/microorganisms7030083

Fijan, S. 2014. Microorganisms with claimed probiotic properties: An overview of recent literature. Int. J. Environ. Res. Public Health. 11:4745-4767. https://doi.org/10.3390/ ijerph110504745

Fijan, S., D. Šulc, \& A. Steyer. 2018. Study of the in vitro antagonistic activity of various single-strain and multi-strain probiotics against Escherichia coli. Int. J. Environ. Res. Public Health. 15:1539. https://doi.org/10.3390/ijerph15071539

Fouhse, J.M., R. T. Zijlstra, \& B. P. Willing. 2016. The role of gut microbiota in the health and disease of pigs. Anim. Front. 6:30-36. https://doi.org/10.2527/af.2016-0031

Fukuda, K., A. Seidavi, Y. Nami, Y. Sun sunyuan, H.-J. Qiu, M. Li, Y. Wang, H. Cui, Y. Li, \& Y. Sun. 2020. Characterization of lactic acid bacteria isolated from the gastrointestinal tract of a wild boar as potential probiotics. Front. Vet. Sci. 7:49. https://doi.org/10.3389/fvets.2020.00049

Georgieva, R., L. Yocheva, L. Tserovska, G. Zhelezova, N. Stefanova, A. Atanasova, A. Danguleva, G. Ivanova, N. Karapetkov, N. Rumyan, \& E. Karaivanova. 2015. Antimicrobial activity and antibiotic susceptibility of Lactobacillus and Bifidobacterium spp. intended for use as starter and probiotic cultures. Biotechnol. Biotechnol. Equip. 29:84-91. https://doi.org/10.1080/13102818.2014.987 450

Henze, L. J., N. J. Koehl, H. Bennett-Lenane, R. Holm, M. Grimm, F. Schneider, W. Weitschies, M. Koziolek, \& B. T. Griffin. 2021. Characterization of gastrointestinal transit and luminal conditions in pigs using a telemetric motility capsule. Eur. J. Pharm. Sci. 156:105627. https://doi. org/10.1016/j.ejps.2020.105627

Hou, C., X. Zeng, F. Yang, H. Liu, \& S. Qiao. 2015. Study and use of the probiotic Lactobacillus reuteri in pigs: A review. J. Anim. Sci. Biotechnol. 6. https://doi.org/10.1186/ s40104-015-0014-3

Hummel, A. S., C. Hertel, W. H. Holzapfel, \& C. M. A. P. Franz. 2007. Antibiotic resistances of starter and probiotic strains of lactic acid bacteria. Appl. Environ. Microbiol. 73:730739. https://doi.org/10.1128/AEM.02105-06

Jomehzadeh, N., H. Javaherizadeh, M. Amin, M. Saki, M. T. S. Al-Ouqaili, H. Hamidi, M. Seyedmahmoudi, \& Z. Gorjian. 2020. Isolation and identification of potential probiotic Lactobacillus species from feces of infants in southwest Iran. Int. J. Infect. Dis. 96:524-530. https://doi. org/10.1016/j.ijid.2020.05.034

Komatsu, T., M. Matsubayashi, N. Murakoshi, K. Sasai, \& T. Shibahara. 2019. Retrospective and histopathological studies of Entamoeba spp. and other pathogens associated with diarrhea and wasting in pigs in Aichi Prefecture, Japan. Jpn. Agric. Res. Q. 53:59-67. https://doi.org/10.6090/ jarq.53.59

Lee, I. K., Y. C. Kye, G. Kim, H. W. Kim, M. J. Gu, J. Umboh, K. Maaruf, S. W. Kim, \& C. H. Yun. 2016. Stress, nutrition, and intestinal immune responses in pigs: A review. 
Asian-Australas. J. Anim. Sci. 29:1075-1082 https://doi. org/10.5713/ajas.16.0118

Novik, G. \& V. Savich. 2020. Beneficial microbiota. Probiotics and pharmaceutical products in functional nutrition and medicine. Microbes Infect. 22:8-18 https://doi.org/10.1016/j. micinf.2019.06.004

Praepanitchai, O., A. Noomhorm, \& A. K. Anal. 2019. Survival and behavior of encapsulated probiotics (Lactobacillus plantarum) in Calcium-Alginate-Soy protein Isolate-Based Hydrogel beads in different processing conditions $(\mathrm{pH}$ and Temperature) and in pasteurized mango juice. Biomed. Res. Int. 2019:9768152. https://doi.org/10.1155/2019/9768152

Ruiz, L., A. Margolles, \& B. Sánchez. 2013. Bile resistance mechanisms in Lactobacillus and Bifidobacterium. Front. Microbiol. 4:396. https://doi.org/10.3389/fmicb.2013.00396

Sayan, H., P. Assavacheep, K. Angkanaporn, \& A. Assavacheep. 2018. Effect of Lactobacillus salivarius on growth performance, diarrhea incidence, fecal bacterial population and intestinal morphology of suckling pigs challenged with F4+ enterotoxigenic Escherichia coli. Asian-Australas. J. Anim. Sci. 31:1308-1314. https://doi.org/10.5713/ajas.17.0746

Schmitt, J. D., L. O. De Fariña, M. Simões, \& L. B. M. Kottwitz. 2018. Evaluation of the probiotic profile of the Lactobacillus acidophilus used in pharmaceutical and food applications. Acta Sci. Health Sci. 40:1-9. https://doi.org/10.4025/actascihealthsci.v40i1.36664

Sharma, C., S. Gulati, N. Thakur, B. P. Singh, S. Gupta, S. Kaur, S. K. Mishra, A. K. Puniya, J. P. S. Gill, \& H. Panwar. 2017. Antibiotic sensitivity pattern of indigenous Lactobacilli isolated from curd and human milk samples. 3 Biotech 7:53. https://doi.org/10.1007/s13205-017-0682-0

Singh, T. P., G. Kaur, S. Kapila, \& R. K. Malik. 2017. Antagonistic activity of Lactobacillus reuteri strains on the adhesion characteristics of selected pathogens. Front. Microbiol. 8:486. https://doi.org/10.3389/fmicb.2017.00486

Śliżewska, K., A. Chlebicz-Wójcik, \& A. Nowak. 2021. Probiotic properties of new Lactobacillus strains intended to be used as feed additives for monogastric animals. Probiotics Antimicrob. Proteins. 13:146-162. https://doi. org/10.1007/s12602-020-09674-3
Sniffen, J. C., L. V. McFarland, C. T. Evans, \& E. J. C. Goldstein. 2018. Choosing an appropriate probiotic product for your patient: An evidence-based practical guide. PLoS ONE 13. https://doi.org/10.1371/journal.pone.0209205

Watkins, C., K. Murphy, E. M. Dempsey, B. P. Murphy, P. W. O'Toole, R. Paul Ross, C. Stanton, \& C. Anthony Ryan. 2018. The viability of probiotics in water, breast milk, and infant formula. Eur. J. Pediatr. 177:867-870. https://doi. org/10.1007/s00431-018-3133-y

Wylensek, D., T. C. A. Hitch, T. Riedel, A. Afrizal, N. Kumar, E. Wortmann, T. Liu, S. Devendran, T. R. Lesker, S. B. Hernández, V. Heine, E. M. Buhl, P. M. D'Agostino, F. Cumbo, T. Fischöder, M. Wyschkon, T. Looft, V. R. Parreira, B. Abt, H. L. Doden, L. Ly, J. M. P. Alves, M. Reichlin, K. Flisikowski, L. N. Suarez, A. P. Neumann, G. Suen, T. De Wouters, S. Rohn, I. Lagkouvardos, E. Allen-Vercoe, C. Spröer, B. Bunk, A. J. Taverne-Thiele, M. Giesbers, J. M. Wells, K. Neuhaus, A. Schnieke, F. Cava, N. Segata, L. Elling, T. Strowig, J. M. Ridlon, T. A. M. Gulder, J. Overmann, \& T. Clavel. 2020. A collection of bacterial isolates from the pig intestine reveals functional and taxonomic diversity. Nat. Commun. 11:6389. https:// doi.org/10.1038/s41467-020-19929-w

Zhang, S., J. H. Oh, L. M. Alexander, M. özçam, \& J. P. Van Pijkeren. 2018. D-Alanyl-D-alanine ligase as a broad-hostrange counterselection marker in Vancomycin-resistant lactic acid bacteria. J. Bacteriol. 200:e00607-17. https://doi. org/10.1128/JB.00607-17

Zhang, Z., H. Zhang, \& T. Liu. 2019. Study on body temperature detection of pig based on infrared technology: A review. Artif. Intell. Agric. 1:14-26. https://doi.org/10.1016/j. aiia.2019.02.002

Zotta, T., E. Parente, \& A. Ricciardi. 2017. Aerobic metabolism in the genus Lactobacillus: impact on stress response and potential applications in the food industry. J. Appl. Microbiol. 122:857-869. https://doi.org/10.1111/jam.13399

Zou, X., M. Weng, X. Ji, R. Guo, W. Zheng, \& W. Yao. 2017. Comparison of antibiotic resistance and copper tolerance of Enterococcus spp. and Lactobacillus spp. isolated from piglets before and after weaning. J. Microbiol. 55:703-710. https://doi.org/10.1007/s12275-017-6241-x 